\title{
Une femme de science exceptionnelle : Mildred S. Dresselhaus
}

\author{
Alfonso San-Miguel(1) (alfonso.san-miguel-fuster@univ-lyon1.fr) \\ et Jean-Louis Sauvajol(2) \\ (1) Institut Lumière-Matière (UMR5306), Université Claude Bernard Lyon 1, \\ 69622 Villeurbanne \\ (2) Laboratoire Charles Coulomb (L2C), Université de Montpellier, CNRS, \\ 34095 Montpellier
}

Première femme à avoir accédé au rang de professeur titulaire au Massachussetts Institute of Technology (MIT), Mildred Spiewak Dresselhaus, ou simplement Millie pour la plupart de ses collègues, est l'exemple d'un engagement passionnel et infatigable pour

la science en général,

la physique en particulier, et pour la reconnaissance de la place des femmes en science. Sa disparition le 20 février 2017 à l'âge de 86 ans, en pleine activité scientifique, a très profondément marqué les communautés de chercheurs engagés dans les nanosciences. Ses contributions à la compréhension du couplage entre propriétés électroniques, vibrationnelles et structurales, dans les différentes formes de carbone sont au centre du développement des nanomatériaux carbonés et de leurs applications.

\section{Des débuts diffıciles}

Le moins qu'on puisse dire est que pour Millie, l'enfant née en 1930 à Manhattan, rien ne laissait présager une brillante carrière scientifique. La scolarité de Mildred Spiewak, fille d'une famille d'immigrés juifs polonais arrivée en Amérique lors de la grande dépression, démarre dans la pauvreté, dans un quartier défavorisé du Bronx. C'est en partie grâce à ses talents de violoniste, qu'à l'âge de seize ans elle obtient une bourse au mérite qui lui permettra d'intégrer Hunter College à Manhattan en 1947. Elle qualifie cet évènement comme le plus important de sa vie, celui qui lui a permis de quitter «le désordre sordide dans lequel j'avais grandi ". Il n'est pas surprenant que Millie ait toujours souligné combien il était important de donner accès à une bonne éducation aux jeunes de toutes conditions.

La vie de Mildred Spiewak prend donc un vrai tournant dès son arrivée à Hunter College. Dans cette institution elle suit les cours de physique moderne de Rosalyn Yalow, physicienne nucléaire et future prix Nobel de médecine (en 1977), qui l'encourage à poursuivre des études de physique. Ses lettres de recommandation lui permettent de passer une année à l’Université de Cambridge, UK, en 1951 et une autre à Harvard. Rosalyn Yalow conseilla et encouragea Millie pendant plusieurs années, un exemple que cette dernière poursuivra durant toute sa carrière à l'égard de nombreuses jeunes physi- ciennes. En 1953, à l'âge de 22 ans, Millie rejoint l'Université de Chicago où elle suivra le cours de physique quantique d'Enrico Fermi, une rencontre fondamentale, dont elle dira qu'elle l'amena à penser comme une physicienne. En 1958, Millie défend dans cette même université sa thèse sur la supraconductivité sous forts champs magnétiques, travail qu'elle a dû mener avec une grande autonomie. En effet, son directeur ne prit connaissance de ses travaux que deux semaines avant sa soutenance car il considérait que, dans les sciences, il n'y avait pas de place pour les femmes. De fait, elle put mener ses travaux de recherche grâce à un excédent d'équipements scientifiques laissés à l'abandon après la Seconde Guerre mondiale et qu'elle rénova pratiquement sans cout additionnel.

\section{Matériaux à base de carbone et spectroscopie Raman}

Millie et son mari, Gene Dresselhaus, physicien théoricien qu'elle rencontra pendant sa thèse, rejoignent en 1960 le Massachusetts Institute of Technology (MIT) à Boston, un des seuls établissements universitaires américains à avoir une politique d'embauche de couples scientifiques. L'intérêt de Millie pour les systèmes carbonés démarre très tôt après son arrivée au Lincoln Laboratory du MIT. En effet, le responsable de son département pense qu'avec la théorie BCS (Bardeen-CooperSchrieffer) de la supraconductivité ce 

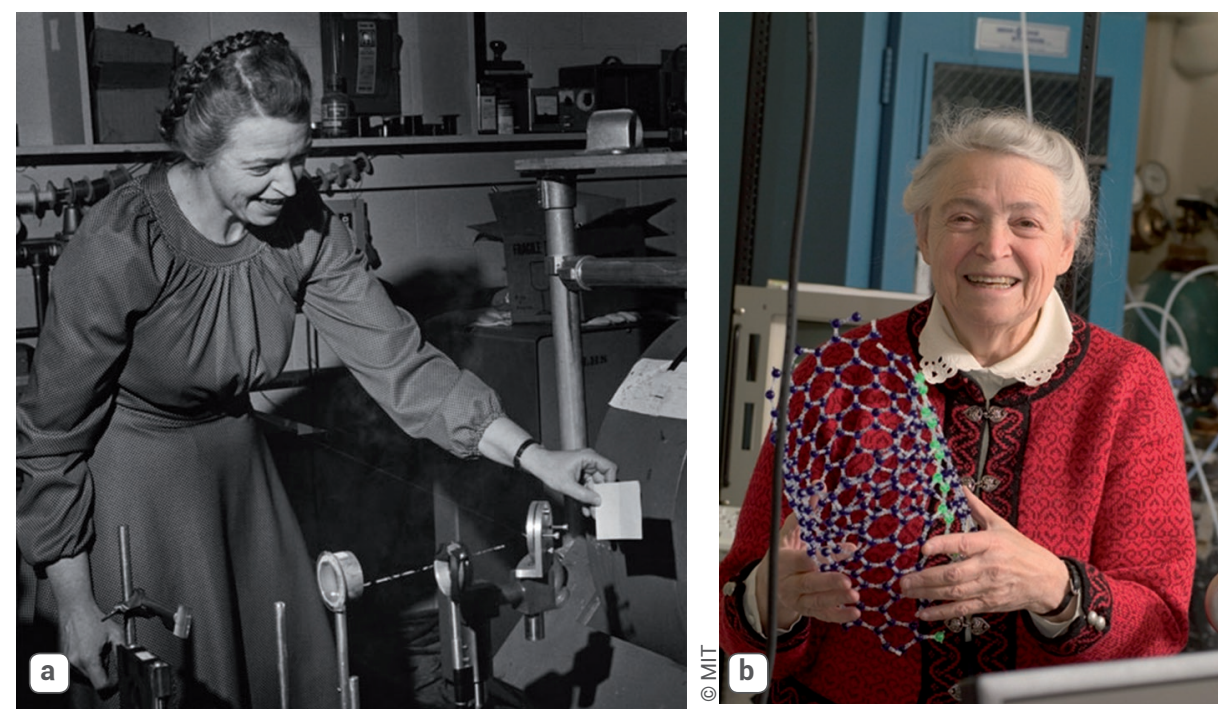

(a) Millie Dresselhaus en train d'aligner une expérience au MIT Lincoln Labs. (Photo : Georgia Litwack). (b) Millie Dresselhaus, en 2006, tient dans ses mains une maquette d'un nanotube de carbone.

domaine de la physique est désormais clos, et lui demande d'explorer d'autres thématiques. Elle choisit de s'orienter dans l'étude des semi-métaux et parmi eux le graphite, matériau considéré à l'époque comme trop complexe en raison de la présence de défauts d'empilement, rendant difficile de disposer de très bons cristaux. De ses propres mots : "contrairement à la physique des semi-conducteurs, la recherche sur le carbone n'était pas très compétitive et obtenir des résultats un jour plus tard n'était pas critique; c'était donc un domaine mieux adapté pour une chercheuse avec une jeune famille. " En effet, Millie et Gene eurent quatre enfants entre 1959 et 1964 , ce qui n'empêcha pas Millie de publier une quinzaine d'articles daurant la même période. Elle identifia ainsi proprement les états des électrons et des trous dans la zone de Brillouin du graphite en utilisant des mesures magnéto-optiques. Millie compte parmi les pionniers de l'utilisation des lasers dans ce type d'expériences. Parmi les techniques expérimentales qu'elle a mises en œuvre durant toute sa carrière, les spectroscopies utilisant des lasers comme sources d'excitation apparaissent comme essentielles à toutes ses recherches.

Ainsi, dès 1970, Millie applique la spectroscopie Raman à l'étude du graphite et de ses composés d'intercalation. Ses travaux pionniers sur l'intercalation des métaux alcalins entre les plans de graphène qui constituent le graphite, ont ouvert la voie, dans un premier temps, au développement des piles alcalines et, plus tard, à celui de la physique du graphène puis, au-delà, à celui des systèmes bidimensionnels (2D).

Millie comprit que la spectroscopie Raman, utilisée alors essentiellement pour caractériser les propriétés vibrationnelles des matériaux, était une technique bien plus puissante. En effet, les processus de résonance liés au couplage photonélectron (autrement dit quand l'énergie du photon est égale ou proche de l'énergie d'une transition électronique), permettent de sonder la structure électronique et d'explorer également les phénomènes de transfert de charge (voir l'encadré, p. 38).

Parmi ses contributions les plus significatives, il faut souligner la première mesure du spectre Raman d'un nanotube de carbone unique (voir l'encadré), qui a motivé différents groupes à étudier les propriétés intrinsèques des nanotubes de carbone individuels. Dans les nanotubes, ce type d'étude n'a été possible que par le caractère résonnant spécifique de l'interaction photon-exciton (paire électron-trou liée), impliquant des états électroniques à forte densité d'états présents dans les nanotubes et conséquence de leur caractère unidimensionnel. Millie développa et utilisa les concepts de Raman résonnant en étudiant des fullerènes, des nanotubes de carbone, du graphène et d'autres systèmes 2D. Au-delà de leur importance dans l'étude des systèmes carbonés, ses travaux ont grandement contribué au développement des spectroscopies optiques moléculaires.

\section{Nanostructuration et thermoélectricité}

Dans les années 1990, en parallèle à ses études sur les nanocarbones, Millie Dresselhaus démarre une nouvelle activité de recherche sur les matériaux thermoélectriques, domaine où elle laissera aussi une empreinte durable [4]. La thermoélectricité est basée sur l'effet Seebeck, le phénomène permettant de générer une différence de potentiel à partir d'une différence de température dans un matériau. Elle est caractérisée par le coefficient Seebeck. À l'origine de la thermoélectricité, on trouve les différences entre les mécanismes de transport thermique et électrique dans les matériaux conducteurs ou semiconducteurs ; le transport thermique est associé principalement aux phonons, les propriétés électriques impliquent les porteurs de charge, électrons ou trous. Millie s'est intéressée à ce sujet après avoir été approchée en 1990 à la fois par la marine américaine et par la marine française qui s'attachaient à l'utilisation de la thermoélectricité pour développer des sous-marins plus silencieux en réduisant le bruit des systèmes de refroidissement. Les travaux de Millie ont permis de montrer le rôle de la nanostructuration et l'intérêt des systèmes de basse dimensionnalité dans l'amélioration des performances des matériaux thermoélectriques. En effet, la nanostructuration permet d'introduire des barrières de diffusion pour le transport électronique ou thermique à travers les interfaces entre nanoparticules, ou entre des nanoparticules 
Millie Dresselhaus ouvrit la voie à la spectroscopie des nano-objets individuels en mesurant pour la première fois le spectre Raman d'un seul nanotube de carbone. La spectroscopie Raman est un processus de diffusion inélastique de la lumière, qui implique un échange d'énergie entre le rayonnement incident d'énergie $\hbar \omega_{\mathrm{i}}$ et la matière (molécules, cristal). Celle-ci peut, en particulier, absorber (ou céder) de l'énergie vibrationnelle au rayonnement incident, conduisant à un rayonnement diffusé $\hbar \omega_{d}$ de plus faible (ou plus forte) énergie. La différence d'énergie entre les photons incident et diffusé, appelée décalage Raman, souvent exprimée en nombre d'onde $\left(\mathrm{cm}^{-1}\right)$, correspond à l'énergie d'un mode de vibration. Les modes collectifs de vibration d'un cristal sont appelés phonons et, en fonction de leur symétrie, certains d'entre eux peuvent être observés en diffusion Raman. L'énergie d'un phonon est notée $\hbar \omega_{\text {ph }}$.

Dans la figure E1a, on montre le spectre Raman, c'est-à-dire l'intensité des photons diffusés en fonction du décalage Raman, d'un seul nanotube semi-conducteur, un cristal unidimensionnel [1, 2]. Les deux panneaux montrent les principaux phonons détectés : le mode de respiration radial spécifique aux nanotubes (RBM pour "radial breathing mode") et les modes optiques tangentiels ou modes $\mathrm{G}$ communs aux carbones dans l'état d'hybridation $\mathrm{sp}^{2}$ [3] (les spectres expérimentaux sont tracés en noir et leur ajustement par des lorentziennes en rouge). Les mouvements atomiques associés sont représentés par les flèches.

Le processus Raman dominant est en réalité un peu plus complexe, car le photon incident est d'abord absorbé par le nanotube semi-conducteur, créant un exciton. Ceci est montré par le diagramme de Feynman de la figure E1b. $\hbar \omega_{i}$, $\hbar \omega_{d}$ et $\hbar \omega_{p h}$ sont respectivement les énergies des photons incident et diffusé et du phonon créé. ex et ex' correspondent à la création et à l'annihilation d'un exciton. Les cercles et le carré symbolisent respectivement les processus d'interaction radiation-électron et électron-phonon.

La section efficace de la diffusion Raman d'un photon est très faible, de l'ordre de $10^{-4}$ de celle d'absorption. C'est le caractère résonnant du processus qui rend donc possible son observation pour un nanotube unique, ce qui se comprend en regardant le schéma E1c. La courbe rose est la densité d'états électroniques (DOS) simplifiée d'un nanotube de carbone semi-conducteur en fonction de l'énergie. On observe les singularités de van Hove (les deux pics de la courbe rose), résultant du caractère unidimensionnel du nanotube. Dans le processus Raman résonnant, équivalent à celui du diagramme de Feynman, l'énergie de l'excitation incidente (flèche bleue) est égale ou proche de l'énergie de transition entre une singularité de van Hove et l'énergie de l'exciton (trait noir horizontal) ; après création du phonon (symbolisé par le point vert), l'état excité est représenté par la ligne noire en tirets ; le système retourne à l'équilibre par émission du photon diffusé (flèche rouge). Le spectre Raman mesure donc ici l'énergie des phonons. L'efficacité du processus est exaltée par la forte disponibilité d'états finaux en raison des fortes densités d'états. Des phénomènes comme les transferts de charge (transferts d'électrons vers ou à partir de molécules adsorbées ou d'une hétérostructure) viennent modifier d'une part la densité d'états électroniques du nanotube et, d'autre part, les forces de liaison des atomes de carbone, affectant ainsi à la fois la position, la largeur et l'intensité des pics Raman. Exploiter le caractère résonnant de la diffusion Raman impose de mesurer des spectres Raman dans une large gamme d'énergies d'excitation, typiquement de 1,2 à 2,7 eV (10 000 à $\left.13700 \mathrm{~cm}^{-1}\right)$, accessible avec les sources laser existantes.

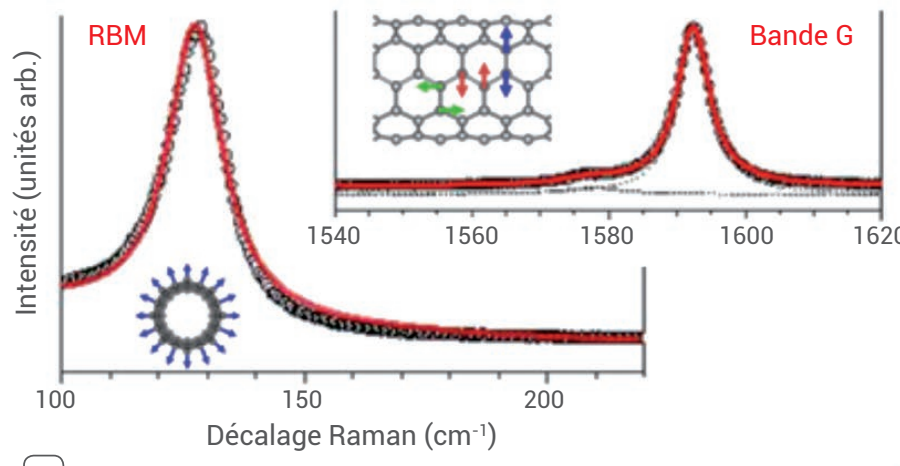

a
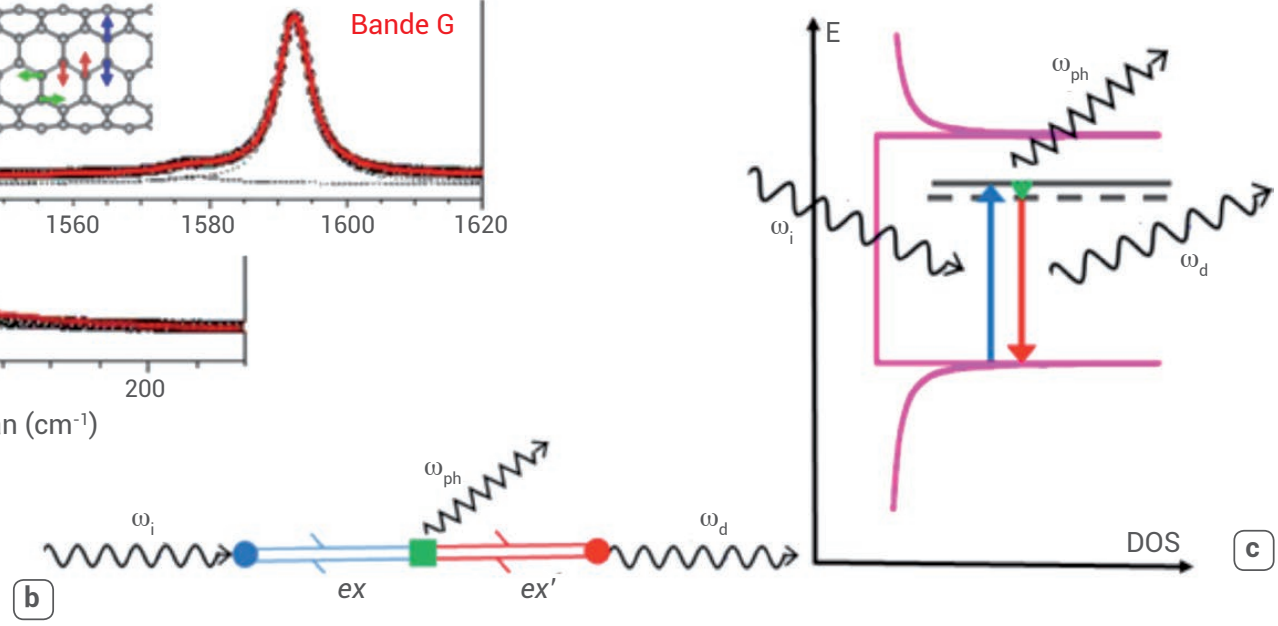

E1. (a) Spectres Raman. (b) Diagramme de Feynman. (c) Schéma du mécanisme de la diffusion Raman. 
et une matrice dans le cas des nanocomposites. En général, la diffusion des phonons par ces frontières est plus importante que celle des porteurs de charge, permettant l'amélioration du facteur de mérite thermoélectrique d'un matériau [5] à travers la nanostructuration. Les effets de dimensionnalité peuvent jouer de leur côté un rôle de différentiation directionnelle dans le transport d'énergie thermique par rapport au transport de charge et constituent également un nouveau levier pour l'optimisation des capacités thermoélectriques d'un matériau donné. Les effets de confinement quantique permettent aussi théoriquement d'obtenir des pics dans la densité d'états électroniques au niveau de Fermi et d'augmenter ainsi le coefficient Seebeck sans sacrifier la conductivité électrique. C'est cet effet, proposé dans l'article fondateur de Hicks et Dresselhaus [6], qui a suscité au début des années 1990 l'engouement de la communauté et le développement d'un nouveau domaine de recherche sur la nanostructuration de matériaux thermoélectriques. Les avancées dans cette thématique, toujours très active à ce jour, ont permis d'améliorer de façon remarquable les propriétés thermoélectriques de ces matériaux, principalement grâce à la réduction de la conductivité thermique. Les contributions de M. Dresselhaus dans ce domaine ont sans aucun doute contribué à sa nomination en 2000 au poste de directrice $d u$ bureau scientifique $d u$ Département de l'Énergie des États-Unis.

\section{Une carrière exceptionnelle et un combat pour la place des femmes en science}

Parmi les honneurs les plus marquants qui sont venus couronner sa carrière scientifique d'exception, nous citerons le prestigieux prix Kavli des nanosciences qu'elle reçut en 2012 pour «ses contributions pionnières dans l'étude des phonons, l'interaction électron-phonon et le transport thermique dans les nanostructures ". M.S. Dresselhaus est actuellement la seule personne à avoir reçu ce prix en tant qu'unique récipiendaire. Nous mentionnerons aussi le prix Enrico Fermi (2012) et la "Presidential Medal of Freedom", la plus haute distinction civile américaine qu'elle reçut en 2014 des mains de Barack Obama. Elle fut également nommée en 1984 présidente de l'American Physical
Society (APS) et elle fut, en 1999, la première femme docteur honoris causa de l'Université Pierre-et-Marie-Curie (type de distinction qu'elle reçut dans plus de trente universités à travers le monde).

Millie était tout autant renommée pour son engagement sans faille pour la promotion des femmes en science et en ingénierie. Elle a ainsi créé, avec une collègue (Emily Wick), le premier "MIT Women's Forum". Elle a aussi œuvré pour créer de meilleures conditions d'accès et de progression pour les jeunes femmes s'engageant dans les disciplines scientifiques. Enfin, elle a travaillé avec la Fondation Carnegie pour contrer la domination masculine aux postes à responsabilités dans la recherche. À sa création en 1990, Millie présida le comité équivalent à la commission « Femmes et Physique " au sein de l'APS, la société sœur américaine de la SFP.

Ses origines polonaises couplées à son infatigabilité au travail - elle arrivait tous les jours au laboratoire à 5 h $30 \ldots$ du lundi au dimanche - pourraient nous faire penser à Marie Curie. Nous pouvons aussi faire un parallèle entre notre " reine du carbone " (comme l'appelaient ses étudiants) et une autre grande scientifique, Rosalind Franklin. Cette dernière contribua grandement dans les années 1940 à la classification des matériaux carbonés, même si elle est plus connue pour sa contribution essentielle à la détermination de la structure de l'ADN, apport qui néanmoins ne fut jamais reconnu par les récipiendaires du prix Nobel pour cette découverte. Comme Rosalind Franklin, Millie Dresselhaus a joué un rôle majeur dans la compréhension des systèmes carbonés et a subi de nombreuses discriminations de genre, notamment dans sa jeunesse.

Au cours de sa longue carrière, aux multiples collaborations de par le monde, Millie Dresselhaus a obtenu des résultats remarquables dans les différentes thématiques qu'elle a abordées et nous laisse un héritage inestimable. Ses qualités scientifiques exceptionnelles, ses contributions de tout premier plan dans la physique nanoscopique, riches de plus de 1700 publications, et son engagement sans faille pour faire progresser la place des femmes en science forcent le respect et lui conferent la reconnaissance unanime de l'ensemble de la communauté scientifique.

\section{Références}

1• H.N. Tran et al., Phys. Rev. B 94 (2016) 075430. Les données de la figure Ela sont adaptées de cet article où, dans les traces de l'héritage de Millie, le caractère excitonique de la résonance Raman est confirmé par l'analyse de son profil.

2. G. Dresselhaus, R. Saito et M.S. Dresselhaus, Physical Properties of Carbon Nanotubes, Imperial College Press (1998).

3. A. Jorio, M.S. Dresselhaus, R. Saito et G. Dresselhaus, Raman Spectroscopy in Graphene Related Systems, Wiley-VCH (2011).

4. Sur ce sujet, voir l'article de Sylvie Hébert, «La recherche de nouveaux matériaux thermoélectriques", paru en octobre 2014 dans le numéro spécial 41 «Femmes et physique » de Reflets de la physique.

5. Le facteur de mérite thermoélectrique, $\mathrm{ZT}$, caractérise les performances thermoélectriques des matériaux. II s'agit, de fait, du produit adimensionnel de la température, $T$, et de $Z$, un facteur de maximisation de l'efficacité thermoélectrique. Il est défini comme $\mathrm{ZT}=\mathrm{TS}^{2} / \rho \kappa$, où $\mathrm{S}$ est le coefficient de Seebeck, $\rho$ la résistivité électrique et $\kappa$ la conductivité thermique du matériau (voir réf. [4] pour plus de détails). Afin d'obtenir un fort ZT, il est nécessaire de maximiser le facteur de puissance $S^{2} / \rho$ et de minimiser la conductivité thermique.

6 L.D. Hicks et M.S. Dresselhaus, Phys. Rev. B 47 (1993) 12727.

\section{Pour en savoir plus}

A. Jorio, "Mildred S. Dresselhaus (1930-2017)", Journal of Raman Spectroscopy, 49 (2018) 13-18.

M. D. Autobiographie, Prix Kavli : www.kavliprize.org/sites/default/ files/\%25nid\%25/autobiagraphies_ attachments/Mildred_Dresselhaus_ Biography_0.pdf] 